\title{
Refined Upper Solution Bound of the Continuous Coupled Algebraic Riccati Equation
}

\author{
Li Wang \\ School of Mathematics and Computational Science, Hunan University of Science and Technology, Xiangtan, \\ Hunan 411201, China
}

Correspondence should be addressed to Li Wang; wanglileigh@163.com

Received 23 May 2020; Accepted 3 July 2020; Published 4 August 2020

Guest Editor: Ramon Costa Castelló

Copyright (c) $2020 \mathrm{Li}$ Wang. This is an open access article distributed under the Creative Commons Attribution License, which permits unrestricted use, distribution, and reproduction in any medium, provided the original work is properly cited.

The continuous coupled algebraic Riccati equation (CCARE) has wide applications in control theory and linear systems. In this paper, by a constructed positive semidefinite matrix, matrix inequalities, and matrix eigenvalue inequalities, we propose a new two-parameter-type upper solution bound of the CCARE. Next, we present an iterative algorithm for finding the tighter upper solution bound of CCARE, prove its boundedness, and analyse its monotonicity and convergence. Finally, corresponding numerical examples are given to illustrate the superiority and effectiveness of the derived results.

\section{Introduction}

Consider the following optimal control of jump linear system described by

$$
\dot{x}(t)=A(r(t)) x(t)+B(r(t)) u(t), \quad x\left(t_{0}\right)=x_{0},
$$

where $x(t) \in R^{n}$ is the plant state and $u(t) \in R^{m}$ is the control vector. $A(r(t)) \in R^{n \times n}, B(r(t)) \in R^{n \times m}$, and $r(t)$ is a finite state Markov jump process on $S=\{1,2, \ldots, s\}$, where $s \geq 2$. The quadratic performance index of system (1) is

$$
J_{u}=E\left\{\int_{0}^{\infty}\left(x^{T}(t) Q(r(t)) x(t)+u^{T}(t) R(r(t)) u(t)\right) \mathrm{d} t \mid x_{0}, r_{0}\right\}
$$

We have $A(r(t))=A_{i}, B(r(t))=B_{i}, Q(r(t))=Q_{i} \epsilon$ $R^{n \times n}$, and $R(r(t))=R_{i} \in R^{m \times m}$ with $Q_{i} \geq 0$ and $R_{i}>0$ when $r(t)=i \in S$. The optimal state feedback controller to minimize the quadratic performance index (2) is $u(t)=$ $-R_{i}^{-1} B_{i}^{T} P_{i} x(t)$, where $P_{i}$ is the symmetric positive semidefinite solution of the continuous coupled algebraic Riccati equation (CCARE):

$$
A_{i}^{T} P_{i}+P_{i} A_{i}-P_{i} B_{i} R_{i}^{-1} B_{i}^{T} P_{i}+\sum_{j \neq i} d_{i j} P_{j}+Q_{i}=0
$$

where $d_{i j}$ are real constants with the properties $d_{i i}<0, d_{i j} \geq$ $0(i \neq j)$, and $\sum_{j \in S} d_{i j}=0$. When $R=I$, the CCARE (3) changes to the following common form:

$$
A_{i}^{T} P_{i}+P_{i} A_{i}-P_{i} B_{i} B_{i}^{T} P_{i}+\sum_{j \neq i} d_{i j} P_{j}+Q_{i}=0 .
$$

CCARE (4) is usually encountered in robust and optimal control [1-8], filter design [9], time-delay systems controller design [10], stability analysis [11-16], etc. And in these fields, it often suffices to estimate the tighter solution bounds of the algebraic Riccati equation rather than get the exact solution. For example, in $[17,18]$, the authors have proposed solution bounds of the continuous algebraic Riccati equation, given their applications of the new bounds in redundant control input systems, and obtained several sufficient conditions to decrease the controller gain of the new systems after the control input extension. In recent years, bounds' estimation of solution for the CCARE has become an attractive topic, and many research approaches have been devoted to this topic. In [19-21], upper matrix bounds for the solution of the CCARE have been presented and iterative algorithms have been proposed to derive tighter upper matrix bounds. And there are many other works for studying the solutions of the CCARE, such as matrix bounds and properties [22-27], 
matrix eigenvalue bounds [28-30], numerical solution [31-33], and the explicit solution [34, 35].

In this paper, we construct a positive semidefinite matrix, propose a new two-parameter-type upper solution bound of the CCARE by matrix inequalities and matrix eigenvalue inequalities. Examples illuminate that the upper bound improves some recent related results. Then, according to the derived solution bound, we give an iterative algorithm, which can guarantee tighter upper solution bound of the CCARE, and prove its boundedness. Subsequently, we analysis its monotonicity and convergence.

Through this paper, let $R^{n \times n}\left(C^{n \times n}\right)$ denote the set of $n \times n$ real (complex) matrices. For $A=\left[a_{i j}\right] \in R^{n \times n}$, the notation $A>0(A \geq 0)$ is used to denote that $\mathrm{A}$ is a symmetric positive definite (semidefinite) matrix. Inequality $A>(\geq) B$ means matrix $(A-B)$ is positive (semi) definite. For $i=1,2, \ldots, n$, let $\lambda_{i}(A)$ be the nonincreasing order eigenvalues of $A$. We assume $A^{T}, A^{-1}$, and $\|A\|_{2}$ are the transpose, the inverse, and the spectral norm of $A$, respectively. $A$ is called a $Z$-matrix if all its off-diagonal elements are nonpositive. It is obvious that any $Z$-matrix $A$ can be written as $s I-B$ with $B$ is nonnegative. A $Z$-matrix $A$ is called an $M$-matrix if $s>\rho(B)$. For two vectors $a=\left(a_{1}, a_{2}, \ldots, a_{n}\right)^{T} \in R^{n}$ and $b=\left(b_{1}, b_{2}\right.$, $\left.\ldots, b_{n}\right)^{T} \in R^{n}, a \geq b$ is equivalent to $a_{i} \geq b_{i}(i=1,2, \ldots, n)$. Symbol $\mu(A)$ represents the matrix measure of $A$ and is defined as $\mu(A)=\lambda_{1}\left(\left(A+A^{T}\right) / 2\right)$.

Lemma 1 (see [36]). For any given positive semidefinite matrix $A \in R^{n \times n}$ and $B \in R^{n \times n}$,

$$
U=B^{T} A+A B \leq 0,
$$

if and only if the matrix $B^{T}+B$ is negative semidefinite.

Lemma 2 (see [37]). Let $A \in R^{n \times n}$ satisfy that $A^{T}+A<0$, and let $P \in R^{n \times n}$ be symmetric. Then,

$$
A^{T} P+P A \leq(<) 0,
$$

if and only if the matrix $P \geq(>) 0$.

Lemma 3 (see [38]). For any symmetric matrix $X$, the following inequality holds:

$$
\lambda_{n}(X) I \leq X \leq \lambda_{1}(X) I .
$$

Lemma 4 (see [38]). Suppose $X, Y \in R^{n \times n}$ are symmetric matrices and $1 \leq i, j \leq n$; then,

$$
\begin{array}{ll}
\lambda_{i+j-1}(X+Y) \leq \lambda_{j}(X)+\lambda_{i}(Y), & i+j \leq n+1, \\
\lambda_{i+j-n}(X+Y) \geq \lambda_{j}(X)+\lambda_{i}(Y), & i+j \geq n+1 .
\end{array}
$$

Lemma 5 (see [39]). If $X \in R^{n \times n}$ is an $M$-matrix, then $X^{-1}$ is nonnegative.

Lemma 6 (see [40]). Let $x=\left(x_{1}, \ldots, x_{n}\right)^{T}, y=\left(y_{1}, \ldots\right.$, $\left.y_{n}\right)^{T} \in R^{n}$ and $x \geq y, A=\left(a_{i j}\right) \in R^{n \times n}$ be nonnegative; then, $A x \geq A y$.
Lemma 7 (see [41]). Let $A, B \in R^{n \times n}$ both be symmetric with $A \geq B$. Then,

$$
\lambda_{i}(A) \geq \lambda_{i}(B),
$$

for all $1 \leq i \leq n$.

Lemma 8 (see [20]). Let $\left\{W_{k}\right\}_{k \in N} \subset R^{n \times n}$ be a given sequence of positive semidefinite matrices. Assume that there exists a positive semidefinite $W_{0} \in R^{n \times n}$ such that for all $k$,

$$
\begin{gathered}
W_{k+1} \leq W_{k}, \\
W_{k} \geq W_{0},
\end{gathered}
$$

and then $\left\{W_{k}\right\}_{k \in N}$ converges to a unique positive semidefinite $\bar{W} \in R^{n \times n}$.

\section{Upper Solution Bounds for the CCARE}

In this section, we will propose new upper matrix bounds of the solution for the CCARE (4), which improve the recent results.

Theorem 1. Let $P_{i}$ be the positive semidefinite solution of the CCARE (4). If there exist some positive definite matrices $K_{i}$, real numbers $n_{i} \geq(1 / 2), \gamma_{i}<0$, and $\gamma_{i}<\beta_{i} \leq-\gamma_{i}$ such that

$$
A_{i}+A_{i}^{T}<\left(B_{i} B_{i}^{T}\right)^{n_{i}} K_{i}+K_{i}\left(B_{i} B_{i}^{T}\right)^{n_{i}}
$$

and suppose $W$ is an M-matrix; then, for $i=1, \ldots, s$,

$$
\begin{aligned}
P_{i} \leq & \frac{1}{-2 \mu\left(\widetilde{A}_{i}\right)}\left(\widetilde{A}_{i}+\gamma_{i} I\right)^{-T}\left(\widetilde{A}_{i}+\beta_{i} I\right)^{T} G_{i}\left(\widetilde{A}_{i}+\beta_{i} I\right)\left(\widetilde{A}_{i}+\gamma_{i} I\right)^{-1} \\
& -\left(\widetilde{A}_{i}+\gamma_{i} I\right)^{-T}\left[\left(\beta_{i}^{2}-\gamma_{i}^{2}\right) \frac{G_{i}}{-2 \mu\left(A_{i}\right)}-\left(\beta_{i}-\gamma_{i}\right) G_{i}\right] \\
& \cdot\left(\widetilde{A}_{i}+\gamma_{i} I\right)^{-1} \equiv P_{u i},
\end{aligned}
$$

where $\widetilde{A}_{i}=A_{i}-\left(B_{i} B_{i}^{T}\right)^{n_{i}} K_{i}, G_{i}=\sum_{j \neq i} d_{i j} \eta_{j} I+Q_{i}+K_{i}\left(B_{i}\right.$
$\left.B_{i}^{T}\right)^{2 n_{i}-1} K_{i}$,

$$
W=\left(\begin{array}{cccc}
1 & -d_{12} r_{1} & \cdots & -d_{1 s} r_{1} \\
-d_{21} r_{2} & 1 & \cdots & -d_{2 s} r_{2} \\
\vdots & & \ddots & \vdots \\
-d_{s 1} r_{s} & -d_{s 2} r_{s} & \cdots & 1
\end{array}\right) \text {, }
$$

$$
\left(\begin{array}{c}
\eta_{1} \\
\eta_{2} \\
\vdots \\
\eta_{s}
\end{array}\right) \stackrel{\text { def }}{=} W^{-1}\left(\begin{array}{c}
\xi_{1} \\
\xi_{2} \\
\vdots \\
\xi_{s}
\end{array}\right)
$$


Complexity

3

$$
\begin{aligned}
r_{i}= & \lambda_{1}\left\{\frac{1}{-2 \mu\left(\widetilde{A}_{i}\right)}\left(\widetilde{A}_{i}+\gamma_{i} I\right)^{-T}\left(\widetilde{A}_{i}+\beta_{i} I\right)^{T}\left(\widetilde{A}_{i}+\beta_{i} I\right)\left(\widetilde{A}_{i}+\gamma_{i} I\right)^{-1}\right. \\
& \left.-\left[\frac{\beta_{i}^{2}-\gamma_{i}^{2}}{-2 \mu\left(\widetilde{A}_{i}\right)}-\left(\beta_{i}-\gamma_{i}\right)\right]\left(\widetilde{A}_{i}+\gamma_{i} I\right)^{-T}\left(\widetilde{A}_{i}+\gamma_{i} I\right)^{-1}\right\} \\
\xi_{i}= & \lambda_{1}\left\{\frac{1}{-2 \mu\left(\widetilde{A}_{i}\right)}\left(\widetilde{A}_{i}+\gamma_{i} I\right)^{-T}\left(\widetilde{A}_{i}+\beta_{i} I\right)^{T}\right. \\
& \cdot\left[Q_{i}+K_{i}\left(B_{i} B_{i}^{T}\right)^{2 n_{i}-1} K_{i}\right]\left(\widetilde{A}_{i}+\beta_{i} I\right)\left(\widetilde{A}_{i}+\gamma_{i} I\right)^{-1} \\
& -\left[\frac{\beta_{i}^{2}-\gamma_{i}^{2}}{-2 \mu\left(\widetilde{A}_{i}\right)}-\left(\beta_{i}-\gamma_{i}\right)\right]\left(\widetilde{A}_{i}+\gamma_{i} I\right)^{-T} \\
& \left.\cdot\left[Q_{i}+K_{i}\left(B_{i} B_{i}^{T}\right)^{2 n_{i}-1} K_{i}\right]\left(\widetilde{A}_{i}+\gamma_{i} I\right)^{-1}\right\} .
\end{aligned}
$$

Proof

$$
\begin{aligned}
& P_{i} B_{i} B_{i}^{T} P_{i}+\varepsilon P_{i}^{2}-K_{i}\left(B_{i} B_{i}^{T}+\varepsilon I\right)^{n_{i}} P_{i} \\
& -P_{i}\left(B_{i} B_{i}^{T}+\varepsilon I\right)^{n_{i}} K_{i}+K_{i}\left(B_{i} B_{i}^{T}+\varepsilon I\right)^{2 n_{i}-1} K_{i} \\
& =\left[P_{i}-\left(B_{i} B_{i}^{T}+\varepsilon I\right)^{n_{i}-1} K_{i}\right]^{T}\left(B_{i} B_{i}^{T}+\varepsilon I\right) \\
& \cdot\left[P_{i}-\left(B_{i} B_{i}^{T}+\varepsilon I\right)^{n_{i}-1} K_{i}\right] \geq 0,
\end{aligned}
$$

where $\varepsilon$ is any sufficiently small positive constant. When $\varepsilon \longrightarrow 0$, from (14), we obtain

$$
\begin{aligned}
0 \leq & P_{i} B_{i} B_{i}^{T} P_{i}-K_{i}\left(B_{i} B_{i}^{T}\right)^{n_{i}} P_{i} \\
& -P_{i}\left(B_{i} B_{i}^{T}\right)^{n_{i}} K_{i}+K_{i}\left(B_{i} B_{i}^{T}\right)^{2 n_{i}-1} K_{i} \equiv S_{i} .
\end{aligned}
$$

And let $\widetilde{Q}_{i}=Q_{i}+\sum_{j \neq i} d_{i j} P_{j}+K_{i}\left(B_{i} B_{i}^{T}\right)^{2 n_{i}-1} K_{i} \geq 0$. According to CARE (4), we obtain

$$
\begin{aligned}
\tilde{A}_{i}^{T} P_{i}+P_{i} \tilde{A}_{i}= & {\left[A_{i}-\left(B_{i} B_{i}^{T}\right)^{n_{i}} K_{i}\right]^{T} P_{i}+P_{i}\left[A_{i}-\left(B_{i} B_{i}^{T}\right)^{n_{i}} K_{i}\right] } \\
= & A_{i}^{T} P_{i}+P_{i} A_{i}-K_{i}\left(B_{i} B_{i}^{T}\right)^{n_{i}} P_{i}-P_{i}\left(B_{i} B_{i}^{T}\right)^{n_{i}} K_{i} \\
= & P_{i} B_{i} B_{i}^{T} P_{i}-Q_{i}-\sum_{j \neq i} d_{i j} P_{j}-K_{i}\left(B_{i} B_{i}^{T}\right)^{n_{i}} P_{i} \\
& -P_{i}\left(B_{i} B_{i}^{T}\right)^{n_{i}} K_{i} \\
= & S_{i}-Q_{i}-\sum_{j \neq i} d_{i j} P_{j}-K_{i}\left(B_{i} B_{i}^{T}\right)^{2 n_{i}-1} K_{i}=S_{i}-\widetilde{Q}_{i} .
\end{aligned}
$$

Let

$$
\begin{aligned}
D_{i}= & \frac{1}{-2 \mu\left(\widetilde{A}_{i}\right)}\left(\widetilde{A}_{i}+\gamma_{i} I\right)^{-T}\left(\widetilde{A}_{i}+\beta_{i} I\right)^{T}\left[\sum_{j \neq i} d_{i j} P_{j}+Q_{i}+K_{i}\left(B_{i} B_{i}^{T}\right)^{2 n_{i}-1} K_{i}\right]\left(\widetilde{A}_{i}+\beta_{i} I\right)\left(\widetilde{A}_{i}+\gamma_{i} I\right)^{-1} \\
& -\left(\widetilde{A}_{i}+\gamma_{i} I\right)^{-T}\left(\beta_{i}^{2}-\gamma_{i}^{2}\right) \frac{\sum_{j \neq i} d_{i j} P_{j}+Q_{i}+K_{i}\left(B_{i} B_{i}^{T}\right)^{2 n_{i}-1} K_{i}}{-2 \mu\left(\widetilde{A}_{i}\right)}\left(\widetilde{A}_{i}+\gamma_{i} I\right)^{-1} \\
& +\left(\widetilde{A}_{i}+\gamma_{i} I\right)^{-T}\left(\beta_{i}-\gamma_{i}\right)\left[\sum_{j \neq i} d_{i j} P_{j}+Q_{i}+K_{i}\left(B_{i} B_{i}^{T}\right)^{2 n_{i}-1} K_{i}\right]\left(\widetilde{A}_{i}+\gamma_{i} I\right)^{-1} \\
= & \left(\widetilde{A}_{i}+\gamma_{i} I\right)^{-T}\left(\widetilde{A}_{i}+\beta_{i} I\right)^{T} \frac{\widetilde{Q}_{i}}{-2 \mu\left(\widetilde{A}_{i}\right)}\left(\widetilde{A}_{i}+\beta_{i} I\right)\left(\widetilde{A}_{i}+\gamma_{i} I\right)^{-1} \\
& -\left(\widetilde{A}_{i}+\gamma_{i} I\right)^{-T}\left(\beta_{i}^{2}-\gamma_{i}^{2}\right) \frac{\widetilde{Q}_{i}}{-2 \mu\left(\widetilde{A}_{i}\right)}\left(\widetilde{A}_{i}+\gamma_{i} I\right)^{-1}+\left(\widetilde{A}_{i}+\gamma_{i} I\right)^{-T}\left(\beta_{i}-\gamma_{i}\right) \widetilde{Q}_{i}\left(\widetilde{A}_{i}+\gamma_{i} I\right)^{-1} .
\end{aligned}
$$

Then, by (16), we have 
4

Complexity

$$
\begin{aligned}
& \tilde{A}_{i}^{T}\left[D_{i}-P_{i}\right]+\left[D_{i}-P_{i}\right] \tilde{A}_{i}=-S_{i}+\widetilde{Q}_{i}+\tilde{A}_{i}^{T} D_{i}+D_{i} \tilde{A}_{i} \\
& =-S_{i}-\frac{1}{\beta_{i}-\gamma_{i}}\left[\left(\widetilde{A}_{i}+\gamma_{i} I\right)^{T} D_{i}\left(\widetilde{A}_{i}+\gamma_{i} I\right)-\left(\widetilde{A}_{i}+\beta_{i} I\right)^{T} D_{i}\left(\widetilde{A}_{i}+\beta_{i} I\right)+\left(\beta_{i}^{2}-\gamma_{i}^{2}\right) D_{i}-\left(\beta_{i}-\gamma_{i}\right) \widetilde{Q}_{i}\right] \\
& =-S_{i}-\frac{1}{\beta_{i}-\gamma_{i}}\left\{\left[\left(\widetilde{A}_{i}+\beta_{i} I\right)^{T} \frac{\widetilde{Q}_{i}}{-2 \mu\left(\widetilde{A}_{i}\right)}\left(\widetilde{A}_{i}+\beta_{i} I\right)-\left(\beta_{i}^{2}-\gamma_{i}^{2}\right) \frac{\widetilde{Q}_{i}}{-2 \mu\left(\widetilde{A}_{i}\right)}+\left(\beta_{i}-\gamma_{i}\right) \widetilde{Q}_{i}\right]\right. \\
& -\left\{\left(\widetilde{A}_{i}+\gamma_{i} I\right)^{-T}\left(\widetilde{A}_{i}+\beta_{i} I\right)^{2 T} \frac{\widetilde{Q}_{i}}{-2 \mu\left(\widetilde{A}_{i}\right)}\left(\widetilde{A}_{i}+\beta_{i} I\right)^{2}\left(\widetilde{A}_{i}+\gamma_{i} I\right)^{-1}\right. \\
& -\left(\widetilde{A}_{i}+\gamma_{i} I\right)^{-T}\left(\widetilde{A}_{i}+\beta_{i} I\right)^{T}\left(\beta_{i}^{2}-\gamma_{i}^{2}\right) \frac{\widetilde{Q}_{i}}{-2 \mu\left(\widetilde{A}_{i}\right)}\left(\widetilde{A}_{i}+\beta_{i} I\right)\left(\widetilde{A}_{i}+\gamma_{i} I\right)^{-1} \\
& \left.+\left(\widetilde{A}_{i}+\gamma_{i} I\right)^{-T}\left(\widetilde{A}_{i}+\beta_{i} I\right)^{T}\left(\beta_{i}-\gamma_{i}\right) \widetilde{Q}_{i}\left(\widetilde{A}_{i}+\beta_{i} I\right)\left(\widetilde{A}_{i}+\gamma_{i} I\right)^{-1}\right\} \\
& +\left(\beta_{i}^{2}-\gamma_{i}^{2}\right)\left[\left(\widetilde{A}_{i}+\gamma_{i} I\right)^{-T}\left(\widetilde{A}_{i}+\beta_{i} I\right)^{T} \frac{\widetilde{Q}_{i}}{-2 \mu\left(\widetilde{A}_{i}\right)}\left(\widetilde{A}_{i}+\beta_{i} I\right)\left(\widetilde{A}_{i}+\gamma_{i} I\right)^{-1}-\left(\widetilde{A}_{i}+\gamma_{i} I\right)^{-T}\left(\beta_{i}^{2}-\gamma_{i}^{2}\right) \frac{\widetilde{Q}_{i}}{-2 \mu\left(\widetilde{A}_{i}\right)}\left(\widetilde{A}_{i}+\gamma_{i} I\right)^{-1}\right. \\
& \left.\left.+\left(\widetilde{A}_{i}+\gamma_{i} I\right)^{-T}\left(\beta_{i}-\gamma_{i}\right) \widetilde{Q}_{i}\left(\widetilde{A}_{i}+\gamma_{i} I\right)^{-1}\right]-\left(\beta_{i}-\gamma_{i}\right) \widetilde{Q}_{i}\right\} \\
& =-S_{i}-\frac{1}{\beta_{i}-\gamma_{i}}\left(\widetilde{A}_{i}+\gamma_{i} I\right)^{-T}\left(\widetilde{A}_{i}+\beta_{i} I\right)^{T}\left[\left(\widetilde{A}_{i}+\gamma_{i} I\right)^{T} \frac{\widetilde{Q}_{i}}{-2 \mu\left(\widetilde{A}_{i}\right)}\left(\widetilde{A}_{i}+\gamma_{i} I\right)\right. \\
& -\left(\widetilde{A}_{i}+\beta_{i} I\right)^{-T}\left(\widetilde{A}_{i}+\gamma_{i} I\right)^{T}\left(\beta_{i}^{2}-\gamma_{i}^{2}\right) \frac{\widetilde{Q}_{i}}{-2 \mu\left(\widetilde{A}_{i}\right)}\left(\widetilde{A}_{i}+\gamma_{i} I\right)\left(\widetilde{A}_{i}+\beta_{i} I\right)^{-1} \\
& -\left(\widetilde{A}_{i}+\beta_{i} I\right)^{T} \frac{\widetilde{Q}_{i}}{-2 \mu\left(\widetilde{A}_{i}\right)}\left(\widetilde{A}_{i}+\beta_{i} I\right)+\left(\beta_{i}^{2}-\gamma_{i}^{2}\right) \frac{\widetilde{Q}_{i}}{-2 \mu\left(\widetilde{A}_{i}\right)}-\left(\beta_{i}-\gamma_{i}\right) \widetilde{Q}_{i}+\left(\beta_{i}^{2}-\gamma_{i}^{2}\right) \frac{\widetilde{Q}_{i}}{-2 \mu\left(\widetilde{A}_{i}\right)} \\
& -\left(\widetilde{A}_{i}+\beta_{i} I\right)^{-T}\left(\beta_{i}^{2}-\gamma_{i}^{2}\right)^{2} \frac{\widetilde{Q}_{i}}{-2 \mu\left(\widetilde{A}_{i}\right)}\left(\widetilde{A}_{i}+\beta_{i} I\right)^{-1} \\
& \left.+\left(\widetilde{A}_{i}+\beta_{i} I\right)^{-T}\left(\beta_{i}^{2}-\gamma_{i}^{2}\right)\left(\beta_{i}-\gamma_{i}\right) \widetilde{Q}_{i}\left(\widetilde{A}_{i}+\beta_{i} I\right)^{-1}\right]\left(\widetilde{A}_{i}+\beta_{i} I\right)\left(\widetilde{A}_{i}+\gamma_{i} I\right)^{-1} \\
& =-S_{i}-\frac{1}{\beta_{i}-\gamma_{i}}\left(\widetilde{A}_{i}+\gamma_{i} I\right)^{-T}\left(\widetilde{A}_{i}+\beta_{i} I\right)^{T}\left\{\left[\left(\widetilde{A}_{i}+\gamma_{i} I\right)^{T} \frac{\widetilde{Q}_{i}}{-2 \mu\left(\widetilde{A}_{i}\right)}\left(\widetilde{A}_{i}+\gamma_{i} I\right)-\left(\widetilde{A}_{i}+\beta_{i} I\right)^{T} \frac{\widetilde{Q}_{i}}{-2 \mu\left(\widetilde{A}_{i}\right)}\left(\widetilde{A}_{i}+\beta_{i} I\right)\right.\right. \\
& \left.+\left(\beta_{i}^{2}-\gamma_{i}^{2}\right) \frac{\widetilde{Q}_{i}}{-2 \mu\left(\widetilde{A}_{i}\right)}-\left(\beta_{i}-\gamma_{i}\right) \widetilde{Q}_{i}\right]-\left(\beta_{i}^{2}-\gamma_{i}^{2}\right)\left(\widetilde{A}_{i}+\beta_{i} I\right)^{-T}\left[\left(\widetilde{A}_{i}+\gamma_{i} I\right)^{T} \frac{\widetilde{Q}_{i}}{-2 \mu\left(\widetilde{A}_{i}\right)}\left(\widetilde{A}_{i}+\gamma_{i} I\right)\right. \\
& \left.\left.-\left(\widetilde{A}_{i}+\beta_{i} I\right)^{T} \frac{\widetilde{Q}_{i}}{-2 \mu\left(\widetilde{A}_{i}\right)}\left(\widetilde{A}_{i}+\beta_{i} I\right)+\left(\beta_{i}^{2}-\gamma_{i}^{2}\right) \frac{\widetilde{Q}_{i}}{-2 \mu\left(\widetilde{A}_{i}\right)}-\left(\beta_{i}-\gamma_{i}\right) \widetilde{Q}_{i}\right]\left(\widetilde{A}_{i}+\beta_{i} I\right)^{-1}\right\}\left(\widetilde{A}_{i}+\beta_{i} I\right)\left(\widetilde{A}_{i}+\gamma_{i} I\right)^{-1} \\
& =-S_{i}+\left(\widetilde{A}_{i}+\gamma_{i} I\right)^{-T}\left(\widetilde{A}_{i}+\beta_{i} I\right)^{T}\left[\left(\widetilde{A}_{i}^{T} \frac{\widetilde{Q}_{i}}{-2 \mu\left(\widetilde{A}_{i}\right)}+\frac{\widetilde{Q}_{i}}{-2 \mu\left(\widetilde{A}_{i}\right)} \widetilde{A}_{i}+\widetilde{Q}_{i}\right)\right. \\
& \left.-\left(\beta_{i}^{2}-\gamma_{i}^{2}\right)\left(\widetilde{A}_{i}+\beta_{i} I\right)^{-T}\left(\widetilde{A}_{i}^{T} \frac{\widetilde{Q}_{i}}{-2 \mu\left(\widetilde{A}_{i}\right)}+\frac{\widetilde{Q}_{i}}{-2 \mu\left(\widetilde{A}_{i}\right)} \widetilde{A}_{i}+\widetilde{Q}_{i}\right)\left(\widetilde{A}_{i}+\beta_{i} I\right)^{-1}\right]\left(\widetilde{A}_{i}+\beta_{i} I\right)\left(\widetilde{A}_{i}+\gamma_{i} I\right)^{-1} \\
& =-S_{i}+\left(\widetilde{A}_{i}+\gamma_{i} I\right)^{-T}\left(\widetilde{A}_{i}+\beta_{i} I\right)^{T}\left[\bar{Q}_{i}-\left(\beta_{i}^{2}-\gamma_{i}^{2}\right)\left(\widetilde{A}_{i}+\beta_{i} I\right)^{-T} \bar{Q}_{i}\left(\widetilde{A}_{i}+\beta_{i} I\right)^{-1}\right]\left(\widetilde{A}_{i}+\beta_{i} I\right)\left(\widetilde{A}_{i}+\gamma_{i} I\right)^{-1},
\end{aligned}
$$


Complexity

5

where $\quad \bar{Q}_{i}=\widetilde{A}_{i}^{T}\left(\widetilde{Q}_{i} /\left(-2 \mu\left(\widetilde{A}_{i}\right)\right)\right)+\left(\widetilde{Q}_{i} /\left(-2 \mu\left(\widetilde{A}_{i}\right)\right)\right) \widetilde{A}_{i}+\widetilde{Q}_{i}$.

According to condition (11), we get $\widetilde{A}_{i}+\widetilde{A}_{i}^{T}=A_{i}+$ $A_{i}^{T}-\left(B_{i} B_{i}^{T}\right)^{n_{i}} K_{i}-K_{i}\left(B_{i} B_{i}^{T}\right)^{n_{i}}<0$; then,

$$
\frac{\widetilde{A}_{i}^{T}}{-2 \mu\left(\widetilde{A}_{i}\right)}+\frac{I}{2}+\frac{\widetilde{A}_{i}}{-2 \mu\left(\widetilde{A}_{i}\right)}+\frac{I}{2}=\frac{\widetilde{A}_{i}+\widetilde{A}_{i}^{T}}{-2 \mu\left(\widetilde{A}_{i}\right)}+I \leq \frac{2 \mu\left(\widetilde{A}_{i}\right) I}{-2 \mu\left(\widetilde{A}_{i}\right)}+I=0 .
$$

Considering $\widetilde{Q}_{i} \geq 0$, using Lemma 1 to (19) yields

$$
\begin{aligned}
\bar{Q}_{i} & =\widetilde{A}_{i}^{T} \frac{\widetilde{Q}_{i}}{-2 \mu\left(\widetilde{A}_{i}\right)}+\frac{\widetilde{Q}_{i}}{-2 \mu\left(\widetilde{A}_{i}\right)} \widetilde{A}_{i}+\widetilde{Q}_{i} \\
& =\left(\frac{\widetilde{A}_{i}^{T}}{-2 \mu\left(\widetilde{A}_{i}\right)}+\frac{I}{2}\right) \widetilde{Q}_{i}+\widetilde{Q}_{i}\left(\frac{\widetilde{A}_{i}}{-2 \mu\left(\widetilde{A}_{i}\right)}+\frac{I}{2}\right) \leq 0 .
\end{aligned}
$$

Substituting (20) into (18) with $\beta_{i}^{2}-\gamma_{i}^{2} \leq 0$ and $S_{i} \geq 0$, we get the right-hand side of (18) is negative semidefinite. Moreover, since $\widetilde{A}_{i}+\widetilde{A}_{i}^{T}<0$, according to Lemma 2, we conclude $D_{i}-P_{i} \geq 0$, that is,

$$
\begin{aligned}
P_{i} \leq D_{i}= & \frac{1}{-2 \mu\left(\widetilde{A}_{i}\right)}\left(\widetilde{A}_{i}+\gamma_{i} I\right)^{-T}\left(\widetilde{A}_{i}+\beta_{i} I\right)^{T} \\
& \cdot\left[\sum_{j \neq i} d_{i j} P_{j}+Q_{i}+K_{i}\left(B_{i} B_{i}^{T}\right)^{2 n_{i}-1} K_{i}\right]\left(\widetilde{A}_{i}+\beta_{i} I\right)\left(\widetilde{A}_{i}+\gamma_{i} I\right)^{-1} \\
& -\left(\widetilde{A}_{i}+\gamma_{i} I\right)^{-T}\left(\beta_{i}^{2}-\gamma_{i}^{2}\right) \frac{\sum_{j \neq i} d_{i j} P_{j}+Q_{i}+K_{i}\left(B_{i} B_{i}^{T}\right)^{2 n_{i}-1} K_{i}}{-2 \mu\left(\widetilde{A}_{i}\right)}\left(\widetilde{A}_{i}+\gamma_{i} I\right)^{-1} \\
& +\left(\widetilde{A}_{i}+\gamma_{i} I\right)^{-T}\left(\beta_{i}-\gamma_{i}\right)\left[\sum_{j \neq i} d_{i j} P_{j}+Q_{i}+K_{i}\left(B_{i} B_{i}^{T}\right)^{2 n_{i}-1} K_{i}\right]\left(\widetilde{A}_{i}+\gamma_{i} I\right)^{-1} .
\end{aligned}
$$

Using Lemma 3 to (21), we obtain

$$
\begin{aligned}
P_{i} \leq & \left\{\frac{1}{-2 \mu\left(\widetilde{A}_{i}\right)}\left(\widetilde{A}_{i}+\gamma_{i} I\right)^{-T}\left(\widetilde{A}_{i}+\beta_{i} I\right)^{T}\left(\widetilde{A}_{i}+\beta_{i} I\right)\left(\widetilde{A}_{i}+\gamma_{i} I\right)^{-1}\right. \\
& \left.-\left[\frac{\beta_{i}^{2}-\gamma_{i}^{2}}{-2 \mu\left(\widetilde{A}_{i}\right)}-\left(\beta_{i}-\gamma_{i}\right)\right]\left(\widetilde{A}_{i}+\gamma_{i} I\right)^{-T}\left(\widetilde{A}_{i}+\gamma_{i} I\right)^{-1}\right\} \sum_{j \neq i} d_{i j} \lambda_{1}\left(P_{j}\right) \\
& +\frac{1}{-2 \mu\left(\widetilde{A}_{i}\right)}\left(\widetilde{A}_{i}+\gamma_{i} I\right)^{-T}\left(\widetilde{A}_{i}+\beta_{i} I\right)^{T}\left[Q_{i}+K_{i}\left(B_{i} B_{i}^{T}\right)^{2 n_{i}-1} K_{i}\right]\left(\widetilde{A}_{i}+\beta_{i} I\right)\left(\widetilde{A}_{i}+\gamma_{i} I\right)^{-1} \\
& -\left[\frac{\beta_{i}^{2}-\gamma_{i}^{2}}{-2 \mu\left(\widetilde{A}_{i}\right)}-\left(\beta_{i}-\gamma_{i}\right)\right]\left(\widetilde{A}_{i}+\gamma_{i} I\right)^{-T}\left[Q_{i}+K_{i}\left(B_{i} B_{i}^{T}\right)^{2 n_{i}-1} K_{i}\right]\left(\widetilde{A}_{i}+\gamma_{i} I\right)^{-1} .
\end{aligned}
$$

Applying Lemma 4 to (22) yields 


$$
\begin{aligned}
\lambda_{1}\left(P_{i}\right) \leq & \lambda_{1}\left\{\frac{1}{-2 \mu\left(\widetilde{A}_{i}\right)}\left(\widetilde{A}_{i}+\gamma_{i} I\right)^{-T}\left(\widetilde{A}_{i}+\beta_{i} I\right)^{T}\left(\widetilde{A}_{i}+\beta_{i} I\right)\left(\widetilde{A}_{i}+\gamma_{i} I\right)^{-1}\right. \\
& \left.-\left[\frac{\beta_{i}^{2}-\gamma_{i}^{2}}{-2 \mu\left(\widetilde{A}_{i}\right)}-\left(\beta_{i}-\gamma_{i}\right)\right]\left(\widetilde{A}_{i}+\gamma_{i} I\right)^{-T}\left(\widetilde{A}_{i}+\gamma_{i} I\right)^{-1}\right\} \sum_{j \neq i} d_{i j} \lambda_{1}\left(P_{j}\right) \\
& +\lambda_{1}\left\{\frac{1}{-2 \mu\left(\widetilde{A}_{i}\right)}\left(\widetilde{A}_{i}+\gamma_{i} I\right)^{-T}\left(\widetilde{A}_{i}+\beta_{i} I\right)^{T}\left[Q_{i}+K_{i}\left(B_{i} B_{i}^{T}\right)^{2 n_{i}-1} K_{i}\right]\left(\widetilde{A}_{i}+\beta_{i} I\right)\left(\widetilde{A}_{i}+\gamma_{i} I\right)^{-1}\right. \\
& \left.-\left[\frac{\beta_{i}^{2}-\gamma_{i}^{2}}{-2 \mu\left(\widetilde{A}_{i}\right)}-\left(\beta_{i}-\gamma_{i}\right)\right]\left(\widetilde{A}_{i}+\gamma_{i} I\right)^{-T}\left[Q_{i}+K_{i}\left(B_{i} B_{i}^{T}\right)^{2 n_{i}-1} K_{i}\right]\left(\widetilde{A}_{i}+\gamma_{i} I\right)^{-1}\right\} .
\end{aligned}
$$

Therefore, from (23), we obtain $\lambda_{1}\left(P_{i}\right)-r_{i} \sum_{j \neq i}$ $d_{i j} \lambda_{1}\left(P_{j}\right) \leq \xi_{i}, i=1,2, \ldots, s$, i.e.,

$$
W x \leq \xi
$$

where $\quad x=\left(\lambda_{1}\left(P_{1}\right), \lambda_{1}\left(P_{2}\right), \ldots, \lambda_{1}\left(P_{s}\right)\right)$ and $\xi=\left(\xi_{1}\right.$, $\left.\xi_{2}, \ldots, \xi_{s}\right)$. As the coefficient matrix $W$ of $(24)$ is an $M$-matrix, $W^{-1}$ is nonnegative in terms of Lemma 5 . Consequently, (24) is equivalent to

$$
x \leq W^{-1} \xi=\eta
$$

by Lemma 6 , where $\eta=\left(\eta_{1}, \eta_{2}, \ldots, \eta_{s}\right)$. Substituting (25) into (22) completes the proof.

Corollary 1. We show that

(1) $P_{i} \leq \frac{1}{-2 \mu\left(\widetilde{A}_{i}\right)}\left[\sum_{j \neq i} d_{i j} \widetilde{\eta}_{j} I+Q_{i}+K_{i}\left(B_{i} B_{i}^{T}\right)^{2 n_{i}-1} K_{i}\right] \equiv P_{u i}^{\prime}$,

(2) $P_{u i} \leq P_{u i}^{\prime}$

where $\tilde{\gamma}_{i}=\left(1 /\left(-2 \mu\left(\widetilde{A}_{i}\right)\right)\right), \quad \widetilde{\xi}_{i}=\left(1 /\left(-2 \mu\left(\widetilde{A}_{i}\right)\right)\right) \lambda_{1}\left[Q_{i}+K_{i}\right.$ $\left.\left(B_{i} B_{i}^{T}\right)^{2 n_{i}-1} K_{i}\right]$,

$$
\begin{gathered}
\left(\begin{array}{c}
\tilde{\eta}_{1} \\
\tilde{\eta}_{2} \\
\vdots \\
\tilde{\eta}_{s}
\end{array}\right) \text { def } \tilde{W}^{-1}\left(\begin{array}{c}
\tilde{\xi}_{1} \\
\tilde{\xi}_{2} \\
\vdots \\
\tilde{\xi}_{s}
\end{array}\right), \\
\tilde{W}=\left(\begin{array}{cccc}
1 & -d_{12} \tilde{\gamma}_{1} & \cdots & -d_{1 s} \tilde{\gamma}_{1} \\
-d_{21} \tilde{\gamma}_{2} & 1 & \cdots & -d_{2 s} \tilde{\gamma}_{2} \\
\vdots & & \ddots & \vdots \\
-d_{s 1} \tilde{\gamma}_{s} & -d_{s 2} \tilde{\gamma}_{s} & \cdots & 1
\end{array}\right),
\end{gathered}
$$

is an M-matrix.

Proof. (1) Since

$$
\begin{gathered}
\widetilde{A}_{i}^{T}\left(\frac{\widetilde{Q}_{i}}{-2 \mu\left(\widetilde{A}_{i}\right)}-P_{i}\right)+\left(\frac{\widetilde{Q}_{i}}{-2 \mu\left(\widetilde{A}_{i}\right)}-P_{i}\right) \widetilde{A}_{i} \\
\quad=-S_{i}+\widetilde{Q}_{i}+\widetilde{A}_{i}^{T} \frac{\widetilde{Q}_{i}}{-2 \mu\left(\widetilde{A}_{i}\right)}+\frac{\widetilde{Q}_{i}}{-2 \mu\left(\widetilde{A}_{i}\right)} \widetilde{A}_{i}
\end{gathered}
$$

Substituting (20) into (28) and combining $S_{i} \geq 0$, we get the right-hand side of (28) is negative semidefinite. Since $\widetilde{A}_{i}+\widetilde{A}_{i}^{T}<0$, according to Lemma 2 , we conclude $\left(\widetilde{Q}_{i} /\left(-2 \mu\left(\widetilde{A}_{i}\right)\right)\right)-P_{i} \geq 0$, that is,

$$
\begin{aligned}
P_{i} & \leq \frac{\widetilde{Q}_{i}}{-2 \mu\left(\widetilde{A}_{i}\right)}=\frac{1}{-2 \mu\left(\widetilde{A}_{i}\right)}\left[\sum_{j \neq i} d_{i j} P_{j}+Q_{i}+K_{i}\left(B_{i} B_{i}^{T}\right)^{2 n_{i}-1} K_{i}\right] \\
& \leq \frac{1}{-2 \mu\left(\widetilde{A}_{i}\right)}\left[\sum_{j \neq i} d_{i j} \lambda_{1}\left(P_{j}\right) I+Q_{i}+K_{i}\left(B_{i} B_{i}^{T}\right)^{2 n_{i}-1} K_{i}\right] .
\end{aligned}
$$

Using Lemma 4 to (29) yields

$$
\begin{aligned}
\lambda_{1}\left(P_{i}\right) \leq & \frac{1}{-2 \mu\left(\widetilde{A}_{i}\right)} \sum_{j \neq i} d_{i j} \lambda_{1}\left(P_{j}\right) \\
& \quad \frac{1}{-2 \mu\left(\widetilde{A}_{i}\right)} \lambda_{1}\left[Q_{i}+K_{i}\left(B_{i} B_{i}^{T}\right)^{2 n-1} K_{i}\right],
\end{aligned}
$$

that is, $\lambda_{1}\left(P_{i}\right)-\tilde{\gamma}_{i} \sum_{j \neq i} d_{i j} \lambda_{1}\left(P_{j}\right) \leq \tilde{\xi}_{i}$, i.e.,

$$
\widetilde{W} x \leq \widetilde{\xi}
$$

where $\widetilde{\xi}=\left(\xi_{1}, \xi_{2}, \ldots, \xi_{s}\right)$. As the coefficient matrix $\widetilde{W}$ of (31) is an $M$-matrix, $\widetilde{W}^{-1}$ is nonnegative in terms of Lemma 5. Consequently, (31) is equivalent to

$$
x \leq \widetilde{W}^{-1} \widetilde{\xi}=\widetilde{\eta},
$$


by Lemma 6 , where $\tilde{\eta}=\left(\tilde{\eta}_{1}, \tilde{\eta}_{2}, \ldots, \tilde{\eta}_{s}\right)$. Substituting (32) into (29), we get $P_{i} \leq P_{u i}^{\prime}$.

(i) First, let us compare $P_{u i}$ with $\left(G_{i} /\left(-2 \mu\left(\widetilde{A}_{i}\right)\right)\right)$ :

(2)

$$
\begin{aligned}
P_{u i}-\frac{G_{i}}{-2 \mu\left(\widetilde{A}_{i}\right)}= & \frac{1}{-2 \mu\left(\widetilde{A}_{i}\right)}\left(\widetilde{A}_{i}+\gamma_{i} I\right)^{-T}\left(\widetilde{A}_{i}+\beta_{i} I\right)^{T} G_{i}\left(\widetilde{A}_{i}+\beta_{i} I\right)\left(\widetilde{A}_{i}+\gamma_{i}\right)^{-1} \\
& -\left(\widetilde{A}_{i}+\gamma_{i} I\right)^{-T}\left[\left(\beta_{i}^{2}-\gamma_{i}^{2}\right) \frac{G_{i}}{-2 \mu\left(\widetilde{A}_{i}\right)}-\left(\beta_{i}-\gamma_{i}\right) G_{i}\right]\left(\widetilde{A}_{i}+\gamma_{i} I\right)^{-1}-\frac{G_{i}}{-2 \mu(\widetilde{A})} \\
= & \left(\widetilde{A}_{i}+\gamma_{i} I\right)^{-T}\left\{\left(\widetilde{A}_{i}+\beta_{i} I\right)^{T} \frac{G_{i}}{-2 \mu(\widetilde{A})}\left(\widetilde{A}_{i}+\beta_{i} I\right)-\left(\beta_{i}^{2}-\gamma_{i}^{2}\right) \frac{G_{i}}{-2 \mu\left(\widetilde{A}_{i}\right)}+\left(\beta_{i}-\gamma_{i}\right) G_{i}\right. \\
& \left.-\left(\widetilde{A}_{i}+\gamma_{i} I\right)^{T} \frac{G_{i}}{-2 \mu(\widetilde{A})}\left(\widetilde{A}_{i}+\gamma_{i} I\right)\right\}\left(\widetilde{A}_{i}+\gamma_{i} I\right)^{-1} \\
= & \left(\beta_{i}-\gamma_{i}\right)\left(\widetilde{A}_{i}+\gamma_{i} I\right)^{-T}\left\{\widetilde{A}^{T} \frac{G_{i}}{-2 \mu(\widetilde{A})}+\frac{G_{i}}{-2 \mu(\widetilde{A})} \widetilde{A}+G_{i}\right\}\left(\widetilde{A}_{i}+\gamma_{i} I\right)^{-1} .
\end{aligned}
$$

Considering $G_{i} \geq 0$, using Lemma 1 to (19) yields

$\widetilde{A}^{T} \frac{G_{i}}{-2 \mu(\widetilde{A})}+\frac{G_{i}}{-2 \mu(\widetilde{A})} \widetilde{A}+G_{i}$

$$
=\left(\frac{\widetilde{A}_{i}^{T}}{-2 \mu\left(\widetilde{A}_{i}\right)}+\frac{I}{2}\right) G_{i}+G_{i}\left(\frac{\widetilde{A}_{i}}{-2 \mu\left(\widetilde{A}_{i}\right)}+\frac{I}{2}\right) \leq 0 .
$$

Substituting (34) into (33) with $\beta_{i}-\gamma_{i}>0$, we get the right-hand side of (33) is negative semidefinite. Thus,

$$
P_{u i} \leq \frac{G_{i}}{-2 \mu\left(\widetilde{A}_{i}\right)} .
$$

(ii) Next, let us compare $G_{i} /\left(-2 \mu\left(\widetilde{A}_{i}\right)\right)$ with $P_{u i}^{\prime}$. For (35), assume $G_{i}=I$; then, (35) changes to

$$
\begin{aligned}
& \frac{1}{-2 \mu\left(\widetilde{A}_{i}\right)}\left(\widetilde{A}_{i}+\gamma_{i} I\right)^{-T}\left(\widetilde{A}_{i}+\beta_{i} I\right)^{T}\left(\widetilde{A}_{i}+\beta_{i} I\right)\left(\widetilde{A}_{i}+\gamma_{i}\right)^{-1} \\
& -\left[\frac{\beta_{i}^{2}-\gamma_{i}^{2}}{-2 \mu\left(\widetilde{A}_{i}\right)}-\left(\beta_{i}-\gamma_{i}\right)\right]\left(\widetilde{A}_{i}+\gamma_{i} I\right)^{-T}\left(\widetilde{A}_{i}+\gamma_{i} I\right)^{-1} \\
& \leq \frac{1}{-2 \mu\left(\widetilde{A}_{i}\right)} I .
\end{aligned}
$$

By Lemma 7 , it is easily to see $r_{i} \leq \tilde{\gamma}_{i}$, then $W \geq \tilde{W}$. As $W$ and $\widetilde{W}$ are $M$-matrix, we obtain

$$
W^{-1} \leq \tilde{W}^{-1} .
$$

On the contrary, for $G_{i}$ of (35), assume $\sum_{j \neq i} d_{i j} \eta_{j} I=0$; then, (35) changes to

$$
\begin{aligned}
& \frac{1}{-2 \mu\left(\widetilde{A}_{i}\right)}\left(\widetilde{A}_{i}+\gamma_{i} I\right)^{-T}\left(\widetilde{A}_{i}+\beta_{i} I\right)^{T}\left[Q_{i}+K_{i}\left(B_{i} B_{i}^{T}\right)^{2 n_{i}-1} K_{i}\right] \\
& \cdot\left(\widetilde{A}_{i}+\beta_{i} I\right)\left(\widetilde{A}_{i}+\gamma_{i} I\right)^{-1} \\
& \quad-\left[\frac{\beta_{i}^{2}-\gamma_{i}^{2}}{-2 \mu\left(\widetilde{A}_{i}\right)}-\left(\beta_{i}-\gamma_{i}\right)\right]\left(\widetilde{A}_{i}+\gamma_{i} I\right)^{-T} \\
& \cdot\left[Q_{i}+K_{i}\left(B_{i} B_{i}^{T}\right)^{2 n_{i}-1} K_{i}\right]\left(\widetilde{A}_{i}+\gamma_{i} I\right)^{-1} \\
& \leq \frac{1}{-2 \mu\left(\widetilde{A}_{i}\right)}\left[Q_{i}+K_{i}\left(B_{i} B_{i}^{T}\right)^{2 n_{i}-1} K_{i}\right] .
\end{aligned}
$$

Applying Lemma 7 to (38), we conclude $\xi_{i} \leq \widetilde{\xi}_{i}$. Thus, we obtain

$$
\eta_{i} \leq \tilde{\eta}_{i},
$$

with (37). Subsequently, we obtain

$$
\frac{G_{i}}{-2 \mu\left(\widetilde{A}_{i}\right)} \leq \frac{1}{-2 \mu\left(\widetilde{A}_{i}\right)}\left[\sum_{j \neq i} d_{i j} \widetilde{\eta}_{j} I+Q_{i}+K_{i}\left(B_{i} B_{i}^{T}\right)^{2 n_{i}-1} K_{i}\right]=P_{u i}^{\prime} .
$$

Lastly, combining (35) with (40), we derive $P_{u i} \leq P_{u i}^{\prime}$.

Remark 1. Due to the different proof methods, it is very hard to compare the upper bounds of Theorem 1 with the parallel results theoretically, and we will present examples to illustrate that our upper bounds are tighter than the recent results for some cases. 


\section{Iterative Algorithm}

According to Section 2, we give an iterative algorithm as following. The algorithm is based on upper bounds (12) from Theorem 1. And we will discuss the following conclusions in the same condition as Theorem 1 .

Algorithm 1. Let $X_{i}^{(0)}=\eta_{i} I, i \in S$, and $\eta_{i}$ is from Theorem 1 . For $k=1,2, \ldots$,

$$
\begin{aligned}
X_{i}^{(k+1)}= & \frac{1}{-2 \mu\left(\widetilde{A}_{i}\right)}\left(\widetilde{A}_{i}+\gamma_{i} I\right)^{-T}\left(\widetilde{A}_{i}+\beta_{i} I\right)^{T} U_{i}^{(k)} \\
& \cdot\left(\widetilde{A}_{i}+\beta_{i} I\right)\left(\widetilde{A}_{i}+\gamma_{i} I\right)^{-1} \\
& -\left(\beta_{i}^{2}-\gamma_{i}^{2}\right)\left(\widetilde{A}_{i}+\gamma_{i} I\right)^{-T} \frac{U_{i}^{(k)}}{-2 \mu\left(\widetilde{A}_{i}\right)}\left(\widetilde{A}_{i}+\gamma_{i} I\right)^{-1} \\
& +\left(\beta_{i}-\gamma_{i}\right)\left(\widetilde{A}_{i}+\gamma_{i} I\right)^{-T} U_{i}^{(k)}\left(\widetilde{A}_{i}+\gamma_{i} I\right)^{-1}
\end{aligned}
$$

where $U_{i}^{(k)}=\sum_{j \neq i} d_{i j} X_{j}^{(k)}+Q_{i}+K_{i}\left(B_{i} B_{i}^{T}\right)^{2 n_{i}-1} K_{i}$.

Next, we will discuss the boundedness of Algorithm 1 and analyse its monotonicity and convergence.

Theorem 2. Let $X_{i}^{(k)}$ be the iteration in Algorithm 1. If the CCARE (4) has positive semidefinite solution $P_{i}$, then

$$
P_{i} \leq X_{i}^{(k)}, \quad k=0,1,2, \ldots
$$

Proof. We proof the result by induction.

(i) From (25), we get $P_{i} \leq \lambda_{1}\left(P_{i}\right) I \leq \eta_{i} I=X_{i}^{(0)}$. Furthermore,

$$
\begin{aligned}
X_{i}^{(1)}= & \frac{1}{-2 \mu\left(\widetilde{A}_{i}\right)}\left(\widetilde{A}_{i}+\gamma_{i} I\right)^{-T}\left(\widetilde{A}_{i}+\beta_{i} I\right)^{T} U_{i}^{(0)} \\
& \cdot\left(\widetilde{A}_{i}+\beta_{i} I\right)\left(\widetilde{A}_{i}+\gamma_{i} I\right)^{-1} \\
& -\left(\beta_{i}^{2}-\gamma_{i}^{2}\right)\left(\widetilde{A}_{i}+\gamma_{i} I\right)^{-T} \frac{U_{i}^{(0)}}{-2 \mu\left(\widetilde{A}_{i}\right)}\left(\widetilde{A}_{i}+\gamma_{i} I\right)^{-1} \\
& +\left(\beta_{i}-\gamma_{i}\right)\left(\widetilde{A}_{i}+\gamma_{i} I\right)^{-T} U_{i}^{(0)}\left(\widetilde{A}_{i}+\gamma_{i} I\right)^{-1},
\end{aligned}
$$

where $U_{i}^{(0)}=Q_{i}+\sum_{j \neq i} d_{i j} X_{j}^{(0)}+K_{i}\left(B_{i} B_{i}^{T}\right)^{2 n_{i}-1} K_{i}=$ $Q_{i}+\sum_{j \neq} i d_{i j} \eta_{i} I+K_{i}\left(B_{i} B_{i}^{T}\right)^{2 n_{i}-1} K_{i}$. According to Theorem 1, we get $P_{i} \leq P_{u i}=X_{i}^{(1)}$.

(ii) Suppose $P_{i} \leq X_{i}^{(k)}$ for all $k \geq 1$, then $\widetilde{Q}_{i}=Q_{i}+$ $\sum_{j \neq i} d_{i j} P_{j}+K_{i}\left(B_{i} B_{i}^{T}\right)^{2 n_{i}-1} K_{i} \leq Q_{i}+\sum_{j \neq i} d_{i j} X_{j}^{(k)}+$ $K_{i}\left(B_{i} B_{i}^{T}\right)^{2 n_{i}-1} K_{i}=U_{i}^{(k)}$. In a similar way to the proof of (18), we have

$$
\begin{aligned}
\widetilde{A}_{i}^{T} & {\left[X_{i}^{(k+1)}-P_{i}\right]+\left[X_{i}^{(k+1)}-P_{i}\right] \widetilde{A}_{i} } \\
& =-\left(\widetilde{A}_{i}^{T} P_{i}+P_{i} \widetilde{A}_{i}\right)+\widetilde{A}_{i}^{T} X_{i}^{(k+1)}+X_{i}^{(k+1)} \widetilde{A}_{i}=-S_{i}+\widetilde{Q}_{i}+\widetilde{A}_{i}^{T} X_{i}^{(k+1)}+X_{i}^{(k+1)} \widetilde{A}_{i} \\
& \leq-S_{i}+U_{i}^{(k)}+\widetilde{A}_{i}^{T} X_{i}^{(k+1)}+X_{i}^{(k+1)} \widetilde{A}_{i} \\
& =-S_{i}-\frac{1}{\beta_{i}-\gamma_{i}}\left[\left(\widetilde{A}_{i}+\gamma_{i} I\right)^{T} X_{i}^{(k+1)}\left(\widetilde{A}_{i}+\gamma_{i} I\right)-\left(\widetilde{A}_{i}+\beta_{i} I\right)^{T} X_{i}^{(k+1)}\left(\widetilde{A}_{i}+\beta_{i} I\right)+\left(\beta_{i}^{2}-\gamma_{i}^{2}\right) X_{i}^{(k+1)}-\left(\beta_{i}-\gamma_{i}\right) U_{i}^{(k)}\right] \\
& =-S_{i}+\left(\widetilde{A}_{i}+\gamma_{i} I\right)^{-T}\left(\widetilde{A}_{i}+\beta_{i} I\right)^{T}\left[\overline{U_{i}^{(k)}}-\left(\beta_{i}^{2}-\gamma_{i}^{2}\right)\left(\widetilde{A}_{i}+\beta_{i} I\right)^{-T} \overline{U_{i}^{(k)}}\left(\widetilde{A}_{i}+\beta_{i} I\right)^{-1}\right]\left(\widetilde{A}_{i}+\beta_{i} I\right)\left(\widetilde{A}_{i}+\gamma_{i} I\right)^{-1},
\end{aligned}
$$

where $\quad \overline{U_{i}^{(k)}}=\widetilde{A}_{i}^{T}\left(U_{i}^{(k)} /\left(-2 \mu\left(\tilde{A}_{i}\right)\right)\right)+\left(U_{i}^{(k)} /\left(-2 \mu\left(\tilde{A}_{i}\right)\right)\right)$ $\tilde{A}_{i}+U_{i}^{(k)}$. Considering $U_{i}^{(k)} \geq 0$, using Lemma 1 to (19) yield

$$
\begin{aligned}
\overline{U_{i}^{(k)}} & =\widetilde{A}^{T} \frac{U_{i}^{(k)}}{-2 \mu(\widetilde{A})}+\frac{U_{i}^{(k)}}{-2 \mu(\widetilde{A})} \widetilde{A}+U_{i}^{(k)} \\
& =\left(\frac{\widetilde{A}_{i}^{T}}{-2 \mu\left(\widetilde{A}_{i}\right)}+\frac{I}{2}\right) U_{i}^{(k)}+U_{i}^{(k)}\left(\frac{\widetilde{A}_{i}}{-2 \mu\left(\widetilde{A}_{i}\right)}+\frac{I}{2}\right) \leq 0 .
\end{aligned}
$$

Substituting (45) into (44) with $\beta_{i}^{2}-\gamma_{i}^{2} \leq 0$ and $S_{i} \geq 0$, we get the right-hand side of (44) is negative semidefinite.
Moreover, since $\widetilde{A}_{i}^{T}+\widetilde{A}_{i}<0$, according to Lemma 2, we get $P_{i} \leq X_{i}^{(k+1)}$. This completes the induction.

Theorem 3. Let $X_{i}^{(k)}$ be the iteration in Algorithm 1. If $P_{u i} \leq \eta_{i} I, i \in S$, where $P_{u i}$ and $\eta_{i}$ are from Theorem 1 , then the sequence $X_{i}^{(k)}$ is monotone decreasing, i.e.,

$$
X_{i}^{(k+1)} \leq X_{i}^{(k)}, \quad k=0,1,2, \ldots
$$

Proof. We proof the result by induction.

(i) $X_{i}^{(1)}=P_{u i} \leq \eta_{i} I=X_{i}^{(0)}$. 
(ii) Suppose $X_{i}^{(k)} \leq X_{i}^{(k-1)}$ for all $k \geq 1$, then

$$
\begin{aligned}
X_{i}^{(k+1)}-X_{i}^{(k)}= & \frac{1}{-2 \mu\left(\widetilde{A}_{i}\right)}\left(\widetilde{A}_{i}+\gamma_{i} I\right)^{-T}\left(\widetilde{A}_{i}+\beta_{i} I\right)^{T}\left(U_{i}^{(k)}-U_{i}^{(k-1)}\right)\left(\widetilde{A}_{i}+\beta_{i} I\right)\left(\widetilde{A}_{i}+\gamma_{i} I\right)^{-1} \\
& -\left(\beta_{i}^{2}-\gamma_{i}^{2}\right)\left(\widetilde{A}_{i}+\gamma_{i} I\right)^{-T} \frac{U_{i}^{(k)}-U_{i}^{(k-1)}}{-2 \mu\left(\widetilde{A}_{i}\right)}\left(\widetilde{A}_{i}+\gamma_{i} I\right)^{-1} \\
& +\left(\beta_{i}-\gamma_{i}\right)\left(\widetilde{A}_{i}+\gamma_{i} I\right)^{-T}\left(U_{i}^{(k)}-U_{i}^{(k-1)}\right)\left(\widetilde{A}_{i}+\gamma_{i} I\right)^{-1} \\
= & \frac{1}{-2 \mu\left(\widetilde{A}_{i}\right)}\left(\widetilde{A}_{i}+\gamma_{i} I\right)^{-T}\left(\widetilde{A}_{i}+\beta_{i} I\right)^{T}\left[\sum_{j \neq i} d_{i j}\left(X_{j}^{(k)}-X_{j}^{(k-1)}\right)\right]\left(\widetilde{A}_{i}+\beta_{i} I\right)\left(\widetilde{A}_{i}+\gamma_{i} I\right)^{-1} \\
& -\left(\beta_{i}^{2}-\gamma_{i}^{2}\right)\left(\widetilde{A}_{i}+\gamma_{i} I\right)^{-T} \frac{\sum_{j \neq i} d_{i j}\left(X_{j}^{(k)}-X_{j}^{(k-1)}\right)}{-2 \mu\left(\widetilde{A}_{i}\right)}\left(\widetilde{A}_{i}+\gamma_{i} I\right)^{-1} \\
& +\left(\beta_{i}-\gamma_{i}\right)\left(\widetilde{A}_{i}+\gamma_{i} I\right)^{-T}\left[\sum_{j \neq i} d_{i j}\left(X_{j}^{(k)}-X_{j}^{(k-1)}\right)\right]\left(\widetilde{A}_{i}+\gamma_{i} I\right)^{-1}
\end{aligned}
$$

$\leq 0$.

Therefore, the proof is complete.

According to Lemma 8, combining Theorems 2 and 3, we get the following conclusion.

Theorem 4. Let $X_{i}^{(k)}$ be the iteration in Algorithm 1 . If $P_{u i} \leq \eta_{i} I, i \in S$, where $P_{u i}$ and $\eta_{i}$ are from Theorem 1 , then the sequence $X_{i}^{(k)}$ is monotone decreasing, and converges to the unique positive semidefinite matrices $\bar{X}_{i}$.

Now, we present examples to illustrate the effectiveness of the main results, and the stopping criterion in Algorithm 1 is set as

$$
\left\|X_{i}^{(k+1)}-X_{i}^{(k)}\right\|_{2}<10^{-8} .
$$

Example 1 (see [42]). Consider the CCARE (4) with

$$
\begin{aligned}
A_{1} & =\left[\begin{array}{cc}
1 & 1 \\
2 & -2
\end{array}\right], \\
A_{2} & =\left[\begin{array}{cc}
-3 & 2 \\
2 & -4
\end{array}\right], \\
s & =\{1,2\}, \\
B_{1} & =\left[\begin{array}{l}
1 \\
0
\end{array}\right], \\
B_{2} & =\left[\begin{array}{l}
2 \\
3
\end{array}\right], \\
Q_{1} & =\left[\begin{array}{ll}
4 & 3 \\
3 & 4
\end{array}\right],
\end{aligned}
$$

$$
\begin{aligned}
& Q_{2}=\left[\begin{array}{ll}
6 & 2 \\
2 & 3
\end{array}\right], \\
& d_{i j}=\left[\begin{array}{cc}
-0.1 & 0.1 \\
0.1 & -0.1
\end{array}\right] .
\end{aligned}
$$

Choose $n_{1}=1, \gamma_{1}=-1, \beta_{1}=1, K_{1}=[2.66,0.9 ; 0.9,1.6]$; $n_{2}=1, \gamma_{2}=-1, \beta_{2}=1$, and $K_{2}=[0.23,0.1 ; 0.1,0.15], \quad$ and by Theorem 1 , we obtain

$$
\begin{aligned}
\eta & =\left[\begin{array}{l}
7.3194 \\
0.9499
\end{array}\right], \\
W & =\left[\begin{array}{cc}
1.0000 & -0.0652 \\
-0.0111 & 1.0000
\end{array}\right], \\
P_{u 1} & =\left[\begin{array}{ll}
6.5085 & 2.1689 \\
2.1689 & 1.5128
\end{array}\right], \\
P_{u 2} & =\left[\begin{array}{ll}
0.8005 & 0.2798 \\
0.2798 & 0.4234
\end{array}\right] .
\end{aligned}
$$

By Corollary 1, we obtain

$$
\begin{aligned}
\widetilde{W} & =\left[\begin{array}{cc}
1.0000 & -0.0652 \\
-0.0111 & 1.0000
\end{array}\right], \\
P_{u 1}^{\prime} & =\left[\begin{array}{ll}
7.2910 & 3.5194 \\
3.5194 & 3.2029
\end{array}\right], \\
P_{u 2}^{\prime} & =\left[\begin{array}{ll}
0.8363 & 0.2777 \\
0.2777 & 0.4849
\end{array}\right] .
\end{aligned}
$$


By Theorem 3.2 in [21], taking $\tilde{a}_{1}=3, \tilde{a}_{2}=0.1$, $T_{i}=\widetilde{a}_{i} I, a_{i}=3$, and $n=1$, we obtain

$$
\begin{aligned}
& \widetilde{P}_{1}=\left[\begin{array}{ll}
8.9463 & 4.9736 \\
4.9736 & 3.5837
\end{array}\right], \\
& \widetilde{P}_{2}=\left[\begin{array}{ll}
1.3337 & 0.5084 \\
0.5084 & 0.6973
\end{array}\right] .
\end{aligned}
$$

By Theorem 2.2 in [42], taking $K_{1}^{\prime}=[2.600$, $0.8950], K_{2}^{\prime}=[0.5900,0.1467], \quad \gamma_{1}^{\prime}=\gamma_{2}^{\prime}=-1$, and $\beta_{2}^{\prime}=\beta_{2}^{\prime}=$ 1 , we obtain

$$
\begin{aligned}
& \bar{P}_{1}=\left[\begin{array}{ll}
7.4634 & 1.8628 \\
1.8628 & 1.7131
\end{array}\right], \\
& \bar{P}_{2}=\left[\begin{array}{ll}
1.0739 & 0.3657 \\
0.3657 & 0.9246
\end{array}\right] .
\end{aligned}
$$

Obviously, we see $P_{u i} \prec P_{u i}^{\prime} \prec \widetilde{P}_{i}$ and $P_{u i} \prec P_{u i}^{\prime} \prec \bar{P}_{i}$, that is, our bounds in Theorem 1 are tighter than [21, 42].

Since

$$
\begin{aligned}
& X_{1}^{(1)}=P_{u 1}=\left[\begin{array}{ll}
6.5085 & 2.1689 \\
2.1689 & 1.5128
\end{array}\right]<7.3194 I=\eta_{1} I=X_{1}^{(0)}, \\
& X_{2}^{(1)}=P_{u 2}=\left[\begin{array}{ll}
0.8005 & 0.2798 \\
0.2798 & 0.4234
\end{array}\right]<0.9499 I=\eta_{2} I=X_{2}^{(0)},
\end{aligned}
$$

then Algorithm 1 is expected to be strictly monotone decreasing and converges to tighter upper bounds. By computation, the upper solution bounds of CCARE (4) are

$$
\begin{aligned}
& X_{1}^{(6)}=\left[\begin{array}{ll}
6.4928 & 2.1746 \\
2.1746 & 1.4956
\end{array}\right], \\
& X_{2}^{(6)}=\left[\begin{array}{ll}
0.7906 & 0.3022 \\
0.3022 & 0.3674
\end{array}\right],
\end{aligned}
$$

after 6 iterations, which are tighter than $X_{1}^{(0)}$ and $X_{2}^{(0)}$, respectively.

Example 2. Consider the CCARE (4) with

$$
\begin{aligned}
& A_{1}=\left[\begin{array}{cc}
1.8 & 1.2 \\
3.2 & -3
\end{array}\right], \\
& A_{2}=\left[\begin{array}{cc}
-2.2 & 1.3 \\
1.4 & -3.1
\end{array}\right], \\
& A_{3}=\left[\begin{array}{cc}
1.5 & 0.6 \\
0.25 & -0.4
\end{array}\right], \\
& B_{1}=\left[\begin{array}{l}
3.6 \\
0.8
\end{array}\right], \\
& B_{2}=\left[\begin{array}{l}
3.6 \\
4.3
\end{array}\right], \\
& B_{3}=\left[\begin{array}{l}
1.5 \\
0.2
\end{array}\right],
\end{aligned}
$$

$$
\begin{aligned}
Q_{1} & =\left[\begin{array}{ll}
6 & 3 \\
3 & 4
\end{array}\right], \\
Q_{2} & =\left[\begin{array}{ll}
8 & 5 \\
5 & 7
\end{array}\right], \\
Q_{3} & =\left[\begin{array}{ll}
5 & 2 \\
2 & 4
\end{array}\right], \\
d_{i j} & =\left[\begin{array}{lll}
-0.2 & 0.1 & 0.1 \\
0.1 & -0.2 & 0.1 \\
0.1 & 0.1 & -0.2
\end{array}\right], \\
R_{1} & =R_{2}=R_{3}=I, \\
s & =\{1,2,3\} .
\end{aligned}
$$

Choosing $\quad n_{1}=1, \gamma_{1}=-2, \beta_{1}=2, K_{1}=[0.675,0.135 ;$ $0.135,0.21], \quad n_{2}=1, \gamma_{2}=-4, \beta_{2}=4, K_{2}=[0.115,0.05 ; 0.05$, $0.25]$, and $n_{3}=1, \gamma_{3}=-2, \beta_{3}=2, K_{3}=[0.93,0.25 ; 0.25$, 0.65 ], by Theorem 1 , we obtain

$$
\begin{aligned}
& \eta=\left[\begin{array}{l}
1.5421 \\
1.1661 \\
7.2392
\end{array}\right], \\
& W=\left[\begin{array}{ccc}
1.0000 & -0.0142 & -0.0142 \\
-0.0145 & 1.0000 & -0.0145 \\
-0.1098 & -0.1098 & 1.0000
\end{array}\right] \text {, } \\
& P_{u 1}=\left[\begin{array}{ll}
1.2524 & 0.4801 \\
0.4801 & 0.6508
\end{array}\right] \text {, } \\
& P_{u 2}=\left[\begin{array}{ll}
1.0065 & 0.2021 \\
0.2021 & 0.7353
\end{array}\right] \text {, } \\
& P_{u 3}=\left[\begin{array}{ll}
6.0565 & 1.7940 \\
1.7940 & 4.2349
\end{array}\right] \text {. }
\end{aligned}
$$

By Corollary 1, we obtain

$$
\begin{aligned}
& P_{u 1}^{\prime}=\left[\begin{array}{ll}
1.9408 & 0.6613 \\
0.6613 & 0.8035
\end{array}\right], \\
& P_{u 2}^{\prime}=\left[\begin{array}{ll}
1.3952 & 0.8388 \\
0.8388 & 1.4212
\end{array}\right], \\
& P_{u 3}^{\prime}=\left[\begin{array}{ll}
8.2740 & 2.9966 \\
2.9966 & 5.1641
\end{array}\right] .
\end{aligned}
$$

By Theorem 3.1 in [21], taking $\tilde{a}_{1}=0.5, \widetilde{a}_{2}=1.6, \widetilde{a}_{3}=$ 1.0, $T_{i}=\widetilde{a}_{i} I, a_{i}=2$, and $n=1$, we obtain 


$$
\begin{aligned}
& \widetilde{P}_{1}=\left[\begin{array}{ll}
4.7453 & 0.4324 \\
0.4324 & 3.1767
\end{array}\right], \\
& \widetilde{P}_{2}=\left[\begin{array}{ll}
2.6529 & 0.5609 \\
0.5609 & 2.8528
\end{array}\right], \\
& \widetilde{P}_{3}=\left[\begin{array}{ll}
8.5305 & 1.2422 \\
1.2422 & 9.4916
\end{array}\right] .
\end{aligned}
$$

By Theorem 2.2 in [42], taking $\gamma_{1}^{\prime}=-0.2, \beta_{1}^{\prime}=0.2, K_{1}^{\prime}=$ $[3.55,2.9], \gamma_{2}^{\prime}=-0.4, \beta_{2}^{\prime}=0.4, K_{2}^{\prime}=[0.11,0.75], \gamma_{3}^{\prime}=-0.68$, $\beta_{3}^{\prime}=0.68$, and $K_{3}^{\prime}=[3.33,1.95]$, we obtain

$$
\begin{aligned}
& \bar{P}_{1}=\left[\begin{array}{ll}
2.8058 & 0.1316 \\
0.1316 & 2.5964
\end{array}\right], \\
& \bar{P}_{2}=\left[\begin{array}{ll}
3.3744 & 0.0925 \\
0.0925 & 3.5902
\end{array}\right], \\
& \bar{P}_{3}=\left[\begin{array}{ll}
8.3780 & 5.2328 \\
5.2328 & 9.8737
\end{array}\right] .
\end{aligned}
$$

Obviously, we see $P_{u i}<P_{u i}^{\prime} \prec \widetilde{P}_{i}$ and $P_{u i}<P_{u i}^{\prime}<\bar{P}_{i}$, that is, our bounds in Theorem 1 are tighter than [21, 42].

Since

$$
\begin{aligned}
& X_{1}^{(1)}=P_{u 1}=\left[\begin{array}{ll}
1.2524 & 0.4801 \\
0.4801 & 0.6508
\end{array}\right]<1.5421 I=\eta_{1} I=X_{1}^{(0)}, \\
& X_{2}^{(1)}=P_{u 2}=\left[\begin{array}{ll}
1.0065 & 0.2021 \\
0.2021 & 0.7353
\end{array}\right]<1.1661 I=\eta_{2} I=X_{2}^{(0)}, \\
& X_{3}^{(1)}=P_{u 3}=\left[\begin{array}{ll}
6.0565 & 1.7940 \\
1.7940 & 4.2349
\end{array}\right]<7.2392 I=\eta_{3} I=X_{3}^{(0)},
\end{aligned}
$$

then Algorithm 1 is expected to be strictly monotone decreasing and converges to tighter upper bounds. By computation, the upper solution bounds of CCARE (4) are

$$
\begin{aligned}
& X_{1}^{(7)}=\left[\begin{array}{ll}
1.2391 & 0.4960 \\
0.4960 & 0.5958
\end{array}\right], \\
& X_{2}^{(7)}=\left[\begin{array}{ll}
0.9809 & 0.2295 \\
0.2295 & 0.6990
\end{array}\right], \\
& X_{3}^{(7)}=\left[\begin{array}{ll}
6.0123 & 1.8695 \\
1.8695 & 4.0732
\end{array}\right],
\end{aligned}
$$

after 7 iterations, which are tighter than $X_{1}^{(0)}, X_{2}^{(0)}$, and $X_{3}^{(0)}$, respectively.

\section{Conclusions}

In this paper, a new two-parameter-type upper solution bound of the CCARE has been proposed. Next, an iterative algorithm for finding the tighter upper solution bound of CCARE has been presented, and its boundedness, monotonicity, and convergence have been proved. Finally, corresponding numerical examples are given to illustrate the superiority and effectiveness of the derived results.

\section{Data Availability}

All data generated or analyzed during this study are included in this article.

\section{Conflicts of Interest}

The authors declare that they have no conflicts of interest.

\section{Acknowledgments}

This work was supported in part by the National Natural Science Foundation for Youths of China (11801164), National Natural Science Foundation of China (11971413), Key Project of National Natural Science Foundation of China (91430213), General Project of Hunan Provincial Natural Science Foundation of China (2015JJ2134), and General Project of Hunan Provincial Education Department of China (15C1320).

\section{References}

[1] M. Basin, J. Rodriguez-Gonzalez, and L. Fridman, "Optimal and robust control for linear state-delay systems," Journal of the Franklin Institute, vol. 344, no. 6, pp. 830-845, 2007.

[2] K. Zhou, J. C. Doyle, and K. Glover, Robust and Optimal Control, Prentice-Hall, Upper Saddle River, NJ, USA, 1996.

[3] Y. Shang, K. Liu, N. Cui, N. Wang, K. Li, and C. Zhang, "A compact resonant switched-capacitor heater for lithium-ion battery self-heating at low temperatures," IEEE Transactions on Power Electronics, vol. 35, no. 7, pp. 7134-7144, 2019.

[4] K. Liu, X. Hu, Z. Wei, Y. Li, and Y. Jiang, "Modified Gaussian process regression models for cyclic capacity prediction of lithium-ion batteries," IEEE Transactions on Transportation Electrification, vol. 5, no. 4, pp. 1225-1236, 2020.

[5] M. Lu, Y. Sun, H. Duan et al., "A working condition recognition method based on multivariable trend analysis for gold-antimony rougher flotation," Minerals Engineering, vol. 156, Article ID 106493, 2020.

[6] M. Lu, D. H. Xie, W. H. Gui, L. H. Wu, C. Y. Chen, and C. H. Yang, "A cascaded recognition method for copper rougher flotation working conditions," Chemical Engineering Science, vol. 175, pp. 220-230, 2018.

[7] B. Sun, C. Yang, Y. Wang, W. Gui, I. Craig, and L. Olivier, "A comprehensive hybrid first principles/machine learning modeling framework for complex industrial processes," Journal of Process Control, vol. 86, pp. 30-43, 2020.

[8] H. Kwakernaak and R. Sivan, Linear Optimal Control Systems, Wiley-Interscience, New York, NY, USA, 1972.

[9] X. Su, P. Shi, L. Wu, and Y.-D. Song, "A novel approach to filter design for TCS fuzzy discrete-time systems with timevarying delay," IEEE Transactions on Fuzzy Systems, vol. 20, no. 6, pp. 1114-1129, 2012.

[10] C.-H. Lee, "Simple stabilizability criteria and memoryless state feedback control design for time-delay systems with timevarying perturbations," IEEE Transactions on Circuits and Systems I: Fundamental Theory and Applications, vol. 45, no. 11, pp. 1211-1215, 1998.

[11] H. Fazelinia, R. Sipahi, and N. Olgac, "Stability robustness analysis of multiple time- delayed systems using "building block" concept," IEEE Transactions on Automatic Control, vol. 52, no. 5, pp. 799-810, 2007. 
[12] Y. He, J.-H. She, and M. Wu, "Delay-dependent stability criteria for linear systems with multiple time delays," IEEE Proceedings-Control Theory and Applications, vol. 153, no. 4, pp. 447-452, 2006.

[13] S.-I. Niculescu, C. E. de Souza, L. Dugard, and J.-M. Dion, "Robust exponential stability of uncertain systems with timevarying delays," IEEE Transactions on Automatic Control, vol. 43, no. 5, pp. 743-748, 1998.

[14] J.-J. Yan, "Robust stability analysis of uncertain time delay systems with delay-dependence," Electronics Letters, vol. 37, no. 2, pp. 135-137, 2001.

[15] S.-S. Wang, B.-S. Chen, and T.-P. Lin, "Robust stability of uncertain time-delay systems," International Journal of Control, vol. 46, no. 3, pp. 963-976, 1987.

[16] L. Wu, X. Su, P. Shi, and J. Qiu, "A new approach to stability analysis and stabilization of discrete-time T-S fuzzy timevarying delay systems," IEEE Transactions on Systems, Man, and Cybernetics, Part B (Cybernetics), vol. 41, no. 1, pp. 273-286, 2011.

[17] Y. Xia, C. Cai, M. Yin, and Y. Zou, "Two new upper bounds of the solution for the continuous algebraic Riccati equation and their application," Science China Information Sciences, vol. 58, no. 5, pp. 1-12, 2015.

[18] J. Liu and L. Wang, "New solution bounds of the continuous algebraic Riccati equation and their applications in redundant control input systems," Science China Information Sciences, vol. 62, no. 10, Article ID 202201, 2019.

[19] R. Davies, P. Shi, and R. Wiltshire, "Upper solution bounds of the continuous and discrete coupled algebraic Riccati equations," Automatica, vol. 44, no. 4, pp. 1088-1096, 2008.

[20] J. Xu and M. Xiao, "On the iterative refinement of matrix upper bounds for the solution of continuous coupled algebraic Riccati equations," Automatica, vol. 49, no. 7, pp. 2168-2175, 2013

[21] J. Liu, Y. Wang, and J. Zhang, "New upper matrix bounds with power form for the solution of the continuous coupled algebraic Riccati matrix equation," Asian Journal of Control, vol. 19, no. 2, pp. 739-747, 2017.

[22] R. Davies, P. Shi, and R. Wiltshire, "New lower matrix bounds for the solution of the continuous algebraic lyapunov equation," Asian Journal of Control, vol. 10, no. 4, pp. 449-455, 2008.

[23] J. Liu, L. Wang, and J. Zhang, "The solution bounds and fixed point iterative algorithm for the discrete coupled algebraic Riccati equation applied to automatic control," IMA Journal of Mathematical Control and Information, vol. 34, no. 4, pp. 1135-1156, 2016.

[24] J. Liu, L. Wang, and J. Zhang, "New matrix bounds and iterative algorithms for the discrete coupled algebraic Riccati equation," International Journal of Control, vol. 90, no. 11, pp. 2326-2337, 2017.

[25] Q. Liu, C. Wang, and Z. Wang, “On Littlewood's boundedness problem for relativistic oscillators with anharmonic potentials," Journal of Differential Equations, vol. 257, no. 12, pp. 4542-4571, 2014.

[26] C.-H. Lee, "An improved lower matrix bound of the solution of the unified coupled Riccati equation," IEEE Transactions on Automatic Control, vol. 50, no. 8, pp. 1221-1223, 2005.

[27] L. Zhou, J. Liu, and L. Zhu, "The closure property of $H$-tensors under the Hadamard product," Journal of Inequalities \& Applications, vol. 2017, no. 1, p. 231, 2017.

[28] J. Liu and J. Zhang, "New upper and lower eigenvalue bounds for the solution of the continuous algebraic Riccati equation," Asian Journal of Control, vol. 16, no. 1, pp. 284-291, 2014.
[29] R. Huang, "A periodic qd-type reduction for computing eigenvalues of structured matrix products to high relative accuracy," Journal of Scientific Computing, vol. 75, no. 3, pp. 1229-1261, 2018.

[30] R. Huang and D. Chu, "Relative perturbation analysis for eigenvalues and singular values of totally nonpositive matrices," SIAM Journal on Matrix Analysis and Applications, vol. 36, no. 2, pp. 476-495, 2015.

[31] P. Benner and J. Saak, "Numerical solution of large and sparse continuous time algebraic matrix Riccati and Lyapunov equations: a state of the art survey," GAMM-mitteilungen, vol. 36, no. 1, pp. 32-52, 2013.

[32] R. Huang, "A qd-type algorithm for computing the generalized singular values of $\mathrm{BF}$ matrices with sign regularity to high relative accuracy," Mathematics of Computation, vol. 89, no. 321, pp. 229-252, 2020.

[33] M. Lu, D. Liu, Y. Deng, L. Wu, Y. Xie, and Z. Chen, "R-K algorithm: a novel dynamic feature matching method of flotation froth," Measurement, vol. 156, Article ID 107581, 2020.

[34] C. Yang, J. Liu, and Y. Liu, "Solutions of the generalized Sylvester matrix equation and the application in eigenstructure assignment," Asian Journal of Control, vol. 14, no. 6, pp. 1669-1675, 2012.

[35] A. J. Rojas, "Explicit solution for a class of discrete-time algebraic Riccati equations," Asian Journal of Control, vol. 15, no. 1, pp. 132-141, 2013.

[36] A. Ostrowski and H. Schneider, "Some theorems on the inertia of general matrices," Journal of Mathematical Analysis and Applications, vol. 4, no. 1, pp. 72-84, 1962.

[37] F. R. Gantmakher, The Theory of Matrices, American Mathematical Soc., Providence, RI, USA, 2000.

[38] D. S. Bernstein, Matrix Mathematics: Theory, Facts, and Formulas with Application to Linear Systems Theory, Princeton University Press, Princeton, NJ, USA, 2005.

[39] A. Berman and R. J. Plemmons, Nonnegative Matrices in the Mathematical Sciences, Society of Indian Automobile Manufacturers (SIAM), Philadelphia, PA, USA, 1994.

[40] J. Liu and J. Zhang, "New upper matrix bounds of the solution for perturbed continuous coupled algebraic Riccati matrix equation," International Journal of Control, Automation and Systems, vol. 10, no. 6, pp. 1254-1259, 2012.

[41] R. A. Horn and C. R. Johnson, Matrix Analysis, Cambridge University Press, Cambridge, UK, 1990.

[42] J. Xu and P. Rajasingam, "New unified matrix upper bound on the solution of the continuous coupled algebraic Riccati equation," Journal of the Franklin Institute, vol. 353, no. 5, pp. 1233-1247, 2016. 